\title{
La sexualité, le genre et l'autre, dans les romans de Michel Houellebecq
}

\author{
ZEINA NAJJAR \\ Université Libanaise \\ 凶ajjarzeina2@gmail.com
}

RÉSUMÉ. Dans cet article nous essayons d'analyser comment Houellebecq racialise les référents de certains concepts tels que : «race ", "culture ", "espèce ", " ethnicité ". Dans ses romans, il décrit l'organisation sociale de la communauté humaine, comme un lieu de tension où se fondent les structures sociales de la société de consommation contemporaine. II peint le paysage de l'imaginaire social de la sexualité et du genre qui est à l'origine de pratiques, mentalités, tendances, fantaisies et imagination, lesquelles nous invitent à déchiffrer un positionnement idéologique qui d'abord, présente en termes d'intempérance, la vie sexuelle dans la société, et ensuite, installe des aspects variés de la discrimination : occidental/ non-occidental, male / femelle.

RESUMEN. En este artículo tratamos de analizar cómo Houellebecq "racializa» los referentes de ciertos conceptos como "raza», "cultura», "especie», "etnia». En sus novelas describe, en efecto, la organización social de la comunidad humana como un lugar de tensiones donde se encuentran las grandes estructuras sociales básicas de la sociedad de consumo contemporánea. Constituye el cuadro de la imaginación social de la sexualidad y del género, siendo el origen de las prácticas sociales, mentalidades,

\section{MOTS-CLÉS :}

sexualité ; genre ; femme ; imaginaire ; autre

PALABRAS CLAVE: sexualidad; género; mujer; fantasía; el otro

\section{Pour citer cet article}

Najjar, Z. (2020). La Sexualité, le Genre et l'Autre, dans les romans de Michel Houellebecq. Hybrida, 1, 171-187. http://doi.org/10.7203/HYBRIDA.1.16876 
tendencias, fantasías e imaginación, que invitan a descifrar un posicionamiento ideológico que, por una parte, presenta en términos de intemperancia la vida sexual en la sociedad, por otra parte, instala diversos aspectos discriminatorios: occidental/no occidental, masculino/ femenino.

ABSTRACT. In this article we try to analyse how Houellebecq "racializes" the referents of certain concepts such as "race", "culture", "species", "ethnicity". In his novels, he describes the social organization of the human community as a place of tension where the basic social structures of the contemporary consumer society are found. He paints a picture of the social imaginary of sexua-

KEYWORDS: sexuality; gender; woman; imagination; other lity and gender, being at the origin of social practices, mentalities, tendencies, fantasies and imagination, which invite us to decipher an ideological position which first, present, in terms of intemperance, the sexual life in the society, and, installs second, some various discriminatory aspects: Occidental/non-occidental, male / female. 


\section{Introduction}

Houellebecq, écrivain contemporain concerné par les grands enjeux du XXI ${ }^{e}$, aime manipuler la provocation, il aborde des thèmes assez controversés, comme la religion, la vie sexuelle, la pornographie. Son ethos ne se soucie guère d'euphémiser les propos et son outrance va jusqu'à des prises de positions ouvertes qui laissent trahir des polarisations idéologiques extrêmes et le font sombrer dans la pure discrimination. Nous essayons de lire ces confrontations idéologiques dans l'intimité de personnages-consciences, dans la mesure où ses romans présentent une opposition idéologique entre deux systèmes antagoniques, celui de l'idéal de justice et de liberté de Mai 68 et celui du néolibéralisme consumériste de la société contemporaine occidentale.

Ses romans se prêtent aisément à être traités dans l'axe de l'hybridation et du métissage. Le «métissage » qui définit généralement tout croisement entre deux cultures, est en concurrence avec le terme « hybridité ", que la sociologie définit comme l'influence mutuelle de cultures différentes en contact les unes avec les autres, notamment dans les domaines artistiques. Cependant les métaphores choisies présupposent l'existence d'un état premier de non-mélange dépassé, caractérisé par la "pureté » des entités en question. Houellebecq opère certains déplacements des subjectivités contemporaines, résultant de la contamination des espaces symboliques (Appaduraï, 2001, p. 69), afin de parvenir à dévoiler un univers qui échappe définitivement à la seule autorité des modes de pensée inspirés de la métaphysique occidentale (Chivallon, 2007, p. 36).

En ce sens, il multiplie les situations qui peuvent porter préjudice à tous ceux dont l'appartenance culturelle diffère de la sienne, et s'affirme ainsi par une esthétique recourant à une littérature " déconcertante " (Chivallon, 2007, p. 36) qui provoque en pensant autrement la société, l'homme et les rapports interhumains. Il aborde les thèmes de genre et de sexualité dans le croisement d'une vision du monde et d'une situation sociale. Le fait de montrer la « mise à mort » de l'intime et de l'intimité, lui a valu dêtrre taxé d'« écrivain-exhibitionniste » (Ippolito, 2001-2002, p. 24).

\section{Contexte de production}

À la suite de l'ébranlement des certitudes par le progrès scientifique, l'univers mental se déstabilise. En renversant l'institution universitaire, pour faire ancrer la rationalité du calcul économique, les soixante-huitards ont bouleversé toutes les institutions de la société traditionnelle, patriarcale et précapitaliste : hédonisme dé- 
mesuré, productivité maximisée, consommation excessive promue par une publicité harcelante. La société prend conscience de l'insignifiance de ses repères, cette césure avec le passé antérieur est d'autant plus brutale, qu'elle n'a pu édifier de présent de substitution.

L'impact de la globalisation se manifeste par le recul de l'hégémonie culturelle française, déployée depuis la Révolution de 1789 sur les autres populations du monde. La société a vaincu l'État et désintégré le peuple premièrement, en le privant de sa mémoire nationale par la déculturation (américanisation) et deuxièmement, en brisant son unité par l'immigration (l'hétérogénéité ethnique). Les concepts tels: hybridation, hybridité, métissage, ressurgissent et constituent le sujet de réflexions et de débats. L'échec de l'institutionnalisation du regroupement familial déclenche à partir des années 1980 les questions religieuses et culturelles au sein des problèmes sociaux. De nouvelles données culturelles surgissent comme la mixité. En effet, les immigrés d'origine islamique vivent sur le sol français le conflit de leur appartenance à une autre culture et subissent de diverses façons la xénophobie.

La faillite des idéologies qui culmine avec la chute du Mur de Berlin en 1989, entraîne l'avènement d'une société de surconsommation, dont les retombées entre autres, l'individualisme et l'hédonisme, se révèlent catastrophiques et sont marquées par le retour à un état primitif : "Selon Daniel Macmillan, ce basculement intervenu dans les civilisations occidentales après 1945 n'était rien d'autre qu'un retour au culte brutal de la force, un refus des règles séculaires lentement bâties au nom de la morale et du droit » (Houellebecq, 2001, p. 27).

La culture marchande établit de nouveaux modes de vie et consacre la dynamique de l'immédiat et du renouvellement perpétuel; droit au bonheur et impatience de satisfaire les désirs poussent les individus à se vautrer dans une sexualité débridée.« Lère de la globalisation est moins modelée par les processus de standardisation et d'homogénéisation que par l'explosion de la diversité, les impératifs de vitesse, la dynamique des flux permanents » (Lipovetsky, 2006, p. 14).

L'outrance devient alors une composante de cette société délivrée prématurément et malgré elle, des normes, sans avoir eu le temps d'en forger d'autres adéquates aux transformations subies de l'époque :

Vivant dans les leurres de la civilisation du désir, les hommes ratent à la fois l'ordre subjectif de leurs existences et l'idéal de justice sociale, en établissant l'imaginaire de la satisfaction complète et immédiate, considéré comme un fétichisme de la croissance des besoins commercialisés. (Lipovetsky, 2006, p. 17) 
Le fétichisme des marques entraîne la disparition des habitus des classes. Le décalage entre être et paraître augmente au fur et à mesure et un schisme au niveau du moi est irrévocable :

les corps sont libres, la misère sexuelle est persistante. Les sollicitations hédonistiques sont omniprésentes : les inquiétudes, les déceptions, les insécurités sociales et personnelles grandissent. Autant d'aspects qui font de la société d'hyperconsommation la civilisation du bonheur paradoxal. (Lipovetsky, 2006, p. 80)

L'acquisition d'une culture fournit la condition nécessaire à la construction d'une personnalité (Todorov, 2008, p. 90) dans cette optique, l'individu fait l'expérience d'une « acculturation » dont le résultat immédiat est une exclusion brutale de la collectivité et par la suite, une crise d'identité. Les individus n'arrivent plus à entamer une identification complète avec l'existence qui est la leur.

La personne n'est rien d'autre que le résultat des innombrables interactions qui jalonnent une vie, autrement dit de l'identité collective: celle-ci est déjà toute faite au moment où l'individu la découvre et elle devient le fondement invisible sur lequel est bâtie son identité...Pour cette raison tout changement qui affecte la culture est vécue comme une atteinte à [son] (mon) intégrité [...] En revanche, ce qui change par la force de circonstances sur lesquelles l'individu n'a aucune prise est perçu comme une dégradation car cela fragilise (notre) [son ] sentiment d'exister. (Todorov, 2008, p. 90)

\section{Une sexualité exacerbée par la surconsommation}

L'univers fictif décrit dans les romans, découle des réalités sociales du début du XXIe siècle. Dans cette société de texte, le personnage prend conscience de son inadaptation. Houellebecq attribue cette aliénation à la réification des relations interhumaines, il insiste sur «la tendance croissante des individus à se percevoir comme des particules isolées ». (Houellebecq, 1998) La communication étant en crise, toutes les relations sont corrompues par l'éphémère et le transitoire et l'amour authentique devient difficile à vivre :

La communication c'est la question de l'autre. Différence quasi ontologique avec l'information. Bien sûr il n'y a pas de message sans destinataire, mais l'information existe néanmoins en soi. Rien de tel, en revanche, pour la communication. Elle n'a du sens qu'au travers de l'existence de l'autre, et de la reconnaissance mutuelle. (Dominique Wolton, 2009, p. 89)

Le personnage houellebecquien soixante-huitard prône l'hédonisme et cherche la satisfaction sexuelle comme condition primordiale au bonheur : 
À part dans l'acte sexuel, il y a peu de moments dans la vie où le corps exulte du simple bonheur de vivre, est rempli de joie par le simple fait de sa présence au monde. (Machado Da Sylva, 2010, pp. 181-187)

Néanmoins, la vulnérabilité du personnage est d'autant plus grande, qu'il n'arrive plus à satisfaire son désir. Soumis à des pulsions émotionnelles il passe dans la sphère de l'involontaire, son identité est modifiée, il n'est plus sujet, mais désormais un non-sujet. (Coquet, 2007, p. 71)

Vivant ses frustrations jusqu'à l'extrême, dans un contexte médiatique qui exacerbe en permanence le désir, il va poursuivre ses fantasmes jusqu'à la démesure : pédophilie, polygamie, échangisme, prostitution, adultère, asexualité totale, meurtre... Bruno et Michel, fils d'une mère hippie vivent brutalement la crise des valeurs traditionnelles de la société libérale, ils ratent complètement leur sexualité par la suite de leur éducation dans la France américanisée de 1968 ; ils sont emblématiques par leur caractère sexuel antinomique, oscillant entre l'abnégation et l'insatisfaction, et dans les deux cas, la négation de l'autre.

L'imaginaire social de l'idéal hédoniste qui embrasse les visées subjectives de plaisir, se conjugue à un imaginaire de divertissement qui s'affirme comme une industrie mondiale, multiplie les occasions d'expérimenter des pratiques sensuelles comblant les insatisfactions. Ces évasions programmées autorisent l'accès à des états d'euphorie ludique (Coquet, 2007, p. 71) déconnectés du réel.

Le désir au sein du couple est maintenu par les fugues aventurières en dehors du mariage [clubs échangistes, adultère, Internet et pornographie (Houellebecq, 2001, p. 3)]. Les nouvelles pratiques de commercialisation et de consommation appellent les hommes et les femmes à être performants; en ce sens, la sexualité devient un service à perfectionner comme d'autres services dans la société : «Au point où nous en sommes, la professionnalisation de la sexualité en Occident est devenue inéluctable.» (Houellebecq, 2001, p. 3)

Paradoxalement, la publicité érotisée mène le désir à son apogée en façonnant un imaginaire amoureux suggestif et stimulant auquel la plupart des gens ne peuvent plus répondre.

et c'était à vrai dire bien plus qu'un simple changement de mode, c'était le retour aux fondamentaux de la nature, de l'attraction sexuelle dans ce qu'elle a de plus élémentaire et de plus brutal, de même lère des mannequins anorexiques était bel et bien terminée, $[\ldots]$ le troisième millénaire à ses débuts revenait, $[\ldots]$ à l'adoration d'un type simple, éprouvé : beauté exprimée dans la plénitude chez la femme, dans la puissance physique chez l'homme. (Houellebecq, 2010, p. 17) 
La recherche des sensations fortes se concrétise dans la quête d'une sexualité, outrée et brutale. L'amour se présente en deux versions différentes, femme et homme, tous les deux faisant défaut et réduits au statut d'objet sexuel. La femme refuse que son image se dilue dans une hypertrophie de l'ego masculin, pour elle, la quête du tendre, débouche sur le vide :

la sexualité a fini par me dégoûter; [...] C'est pénible, à la fin, d'être considérée comme du bétail interchangeable-même si je passais pour une belle pièce, parce que j'étais esthétiquement irréprochable, et qu'ils étaient fiers de m’emmener au restaurant... je crois que j'accordais trop d'importance à l'amour. Je me donnais trop facilement, les hommes me laissaient tomber dès qu'ils étaient arrivés à leurs fins, et j'en souffrais. (Houellebecq, 1998, p. 3)

Les hommes de leur côté, déçus par leurs partenaires sexuelles incompréhensives, optent pour des services payables de la prostitution ou se satisfont dans l'acte plus narcissique de la masturbation :

C'est vraiment rare, maintenant, les femmes qui éprouvent du plaisir, et qui ont envie d'en donner. Séduire une femme qu'on ne connaît pas, baiser avec elle, c'est surtout devenu une source de vexations et de problèmes. [...] on conçoit que les hommes puissent préférer s'éviter beaucoup de soucis en payant une petite somme. Dès qu'ils ont un peu d'âge et d'expérience, ils préfèrent éviter l'amour; ils trouvent plus simple d'aller voir les putes... Il y a aussi tous ceux qui trouvent plus simple de se branler sur Internet, ou en regardant des pornos. (Houellebecq, 2001, p. 36-37)

Dans les milieux de travail, se tissent des relations qui inscrivent des rapports homme / femme en situation de dominant / dominé. En effet, tous les personnages mâles tirent profit de leur statut professionnel par abus de pouvoir : Bruno désire et avance des propositions à ses élèves au lycée (Houellebecq, 1998), Jean-Yves couche avec la baby-sitter de son fils (Houellebecq, 2001), «Daniel 1» profite de l'engouement des femmes attirées par sa célébrité ((Houellebecq, 2005), François exploite prodigieusement son grade de professeur à l'université pour coucher avec ses jeunes étudiantes (Houellebecq, 2015).

Par ailleurs, l'égalité n'est plus différenciation mais identification, la traditionnelle opposition du masculin et du féminin s'estompe. Jean-Pierre Pernaut, le célèbre acteur médiatique avoue son homosexualité en direct devant les caméras de France 2 (Houellebecq, 2010, p. 20), son « outing » déclenche et ancre la visibilité sociale de la question homosexuelle. Les milieux culturels « people » des prix littéraires, rassemblent une foule disparate dans ses expositions des moules de sexes féminin, des actrices porno se font écrivaines. La société du paraître inscrit elle-même son voyeurisme et son outrance. 
Enfin plus qu'une affaire d'échange où le colonisé revend le plaisir au colonisateur, le Tiers-monde, qui résiste mal à la commercialisation, vient combler le manque communicationnel dont souffre l'Occident. Michel symbolise la misère sexuelle des Occidentaux appauvris qui achètent la jouissance asiatique ou latine à bon marché (Houellebecq, 2001).

Cette déréliction où les êtres d'exception deviennent «ultra-people » où l'éthique d'une vie est réduite à une performance $[. .$.$] où le visuel est le seul critère de sélection.$ (Et où) tout est servi avec un point d'exclamation afin de compromettre l'émotion. (Trétiack \& Sirvem, 2007)

\subsection{Le corps mythifié diabolisé}

Dans un siècle marqué par l'individualité et le retour de l'intime, il n'est pas étonnant que le corps devienne un enjeu littéraire majeur. Soulignons tout d'abord, que le corps comme lieu d'inscription de la socialité, expérimente son plaisir par le plaisir d'un autre. Dans les romans d'Houellebecq, la défaillance de ce rapport à l'autre mine le plaisir du personnage, qui désormais n'est plus capable de don, et le réduit à un plaisir solitaire: «lorsque l'affaissement cette fois rédhibitoire de ses chairs ne la conduise à une solitude définitive »(Houellebecq, 2015, p. 23).

La sexualité est vécue par la peur de la finitude du corps en faisant apparaitre l'opacité du corps infranchissable de l'autre :

Lorsque la sexualité disparaît, c'est le corps de l'autre qui apparaît, dans sa présence vaguement hostile ; ce sont les bruits, les mouvements, les odeurs ; et la présence même de ce corps qu'on ne peut plus toucher, ni sanctifier par le contact, devient peu à peu une gêne ; tout cela, malheureusement, est connu. La disparition de la tendresse suit toujours de près celle de l'érotisme. (Houellebecq, 2015, p. 23)

Dans cette frénésie, le corps se rebelle contre l'individu qui le hante. Le personnage continue à être dévoré par le désir et ne se ressent plus en harmonie avec son corps qui cesse d'être un objet de désir. Le corps devient lieu du fugace et se mue en source de déception et en incarnation emblématique de l'impuissance et de la finitude :

ce n'est pas seulement sur le plan émotionnel quelle avait morflé, son corps avait subi des dommages irréparables, ses fesses et ses seins nétaient plus que des surfaces de chair amaigries, réduites, flasques et pendantes, elle ne pouvait plus, ne pourrait jamais plus être considérée comme un objet de désir. (Houellebecq, 2015, p. 22)

La comédie de l'amour physique une fois terminée, le corps se rabat sur d'autres fonctions, c'est ainsi, que les vieux se replient sur les plaisirs gustatifs par une gloutonnerie compensatoire. Pourtant, le corps ayant sa propre date de péremption 
leur ôte même, ce simple plaisir : "dans la plupart des cas les jouissances gustatives s'éteignent irrémédiablement, comme tout le reste. Demeurent les troubles digestifs, et le cancer de la prostate » (Houellebecq, 2010, p. 23).

Les vieux recourent à la prostitution, et cherchent le bonheur, surtout, dans "des corps fermes, souples et sans défaut» (Houellebecq, 2015, p. 23). Les vieilles femmes de leur côté, recherchent le sexe aux centres de loisir New Age et s'engagent dans des chirurgies antiâges. D'autres fois, le corps subit les séquelles du néolibéralisme dévastateur, spécifiquement dans les pays du Tiers-monde, il devient marchandise commercialisée : «Pauvre peuple cubain... prononça-t-il après un long silence. Ils n’ont plus rien à vendre, à l'exception de leurs corps » (Houellebecq, 2001, p. 61).

Cependant, cette quête du corps jeune régénérateur dépasse l'imaginaire social contemporain occidental et rejoint un imaginaire universel, en effet, l'imaginaire du corps jeune qui ranime un corps refroidi et figé par la vieillesse, existe déjà dans La Bible, lorsqu'on cherche une jeune fille vierge au vieux roi David pour le réchauffer ${ }^{1}$.

\subsection{La violence de la sexualité}

Dans les années soixante, les imaginaires sociaux s'imprègnent de la révolution de Woodstock et celle des minorités qui se manifeste en Amérique, par les mouvements noirs, féministes et gays. Les romans de Houellebecq décrivent la violence vécue et imposée et retracent la célébration de toutes les formes de sexualité, en vue de prouver l'impact dévastateur de la culture américaine. Coincé dans cet espace conflictuel de désillusion, le personnage s'obstine à dévoiler la perversité de l'hédonisme prêché par Mai 68 et surtout, la dépravation du mouvement hippie (Houellebecq, 2005, p. 84).

C'est ainsi que, quelle que soit l'altérité dans la relation interpersonnelle, elle est présentée, comme oscillant entre deux extrêmes : l'homosexualité, l'échangisme, le sadomasochisme, la pédophilie, et d'autres actes louches comme les meurtres initiatiques, les viols, les avortements, les suicides, les rituels ésotériques, hérétiques et scatologiques. Dans les clubs sado-maso la musique se superpose aux hurlements des partenaires torturés, la violence verse dans l'outrance à l'état pur. Christine en relatant ses souvenirs de jeunesse, raconte comment la culture américaine a ravagé la vie de David Di Meola, devenu chef de secte sexuelle (Houellebecq, 2005, pp. 169-170) ido-

1 La Bible: 1er livre des Rois: Verset 2. Ses serviteurs lui dirent: Que l'on cherche pour mon seigneur le roi une jeune fille vierge; qu'elle se tienne devant le roi, qu'elle le soigne, et qu'elle couche dans son sein; et mon seigneur le roi se réchauffera. Verset 3. On chercha dans tout le territoire d'Israël une fille jeune et belle, et on trouva Abischag, la Sunamite, que l'on conduisit auprès du roi. 
lâtrant Mick Jagger ${ }^{2}$ : l'image du mal impuni (Houellebecq, 2005, p. 171). Influencé par le satanisme de Charles Manson ${ }^{3}$, il s'implique dans des actes de violence sexuelle et de meurtre : « bébés démembrés par des ciseaux, yeux arrachés, éjaculation dans des orbites humains crevés » (Houellebecq, 2005, p. 83).

\section{Le genre inférieur : la démythification de la femme}

Houellebecq impose sa vision du monde. Il s'agit notamment, de certaines notions d'une idéologie qui se révèle spécifiquement sectaire et se déploie dans des distinctions hiérarchisées, comme le sexisme et le machisme. Dans cette optique, l'idéologie se fonde sur l'idée de la supériorité des mâles, de l'homme sur la femme, au nom d'une supériorité biologique. Houellebecq, cherche à s'affirmer contre la femme en utilisant la violence verbale, la dévalorisation, en la nommant, la représentant et la caractérisant négativement ; un choix qui établit incontestablement, le rapport dominant/dominé.

La grossièreté des choix lexicaux en effet, entraine une outrance extrême ${ }^{4}$ :

- des termes vulgaires et grossiers :

«Pouffiasse » PE, p. 44 prostituée

«Pétasses » PE, p. 44 pour désigner : femme vulgaire

«Gouine»P,p. 89 nf: femme lesbienne

«Salope»PI, p. 156 femme de mauvaise vie

- des métaphores grossières :

«Beaucoup de minijupes, de bustiers échancrés ; bref, de la chair fraiche ». EDL, p. 111

« racler deux minettes » EDL, p. 28 frotter rudement deux jeunes filles

«(de ce type qu'on appelle communément bouche à pipes)» PI, p. 156

2 Mick Jagger a tué Brian Jones et l'a remplacé à la tête des Rolling Stones.

3 Ayant un traumatisme psychique à la suite d'une agression sexuelle à lécole, plusieurs fois incarcéré, marié et divorcé avec un fils, proxénète et intéressé par la scientologie. En 1967, il fonde un groupe hippie,«la Famille», formée essentiellement de jeunes femmes et se présente comme une réincarnation du Christ. Le groupe vit de vols de trafics de drogues et de prostitution. En associant des extraits de la Bible à des textes de l'album blanc des Beatles, Manson conçoit une prophétie, selon laquelle les Noirs vont dominer les Blancs et lui demandent de diriger la nation. Il demande en 1969 à sa communauté de commettre des assassinats dans les beaux quartiers de Los Angeles, meurtres dont il voulait ensuite faire accuser les Noirs. https://www.biography.com/crime-figure/charles-manson

${ }^{4}$ Abréviations: EDL: Extension du domaine de la lutte, P: Plateforme, PI: La possibilité d'une île, PE: Les Particules élémentaires, CT: La carte et le territoire, S: Soumission. 
La femme perd toute valeur humaine, elle n'est pas considérée comme une personne entière et autonome, elle est plutôt vue comme une extension du corps mâle et un accessoire de plaisir. Un sexisme criard se traduit fréquemment dans la synecdoque qui réduit la femme à ces bouts de corps : « des seins, une touffe » (Houellebecq, 2005, p. 413), « des orifices adéquats » (Houellebecq, 2005, p. 35).

Déshumanisée, la femme, devient dans tous les cas un objet à consommer : « un morceau de sucre » (Houellebecq, 2005, p. 413); cette chosification verse dans l'outrance lorsque la femme est doublement discriminée, socialement inférieure (la domination) et biologiquement moindre (femelle): « il y avait aussi les femmes de ménage que je pouvais essayer, certaines étaient tout à fait potables »(Houellebecq, 2005, p. 399).

Par le jeu subtil de mots, la femme instrumentalisée, rime avec un appareil électroménager: " les derniers vacanciers vont repartir ; avec eux les derniers seins, les dernières touffes; les derniers micro-mondes accessibles » (Houellebecq, 2005, p. 423).

La femme est dépourvue de toute densité humaine, d'abord, elle est une espèce différente par référence à Aristote :

En circulant entre les linéaires il songeait que, si l'on en croit Aristote, une femme de petite taille appartient à une espèce différente du reste de l'humanité. Un petit homme me semble encore un homme, écrit le philosophe, mais une petite femme me semble appartenir à une nouvelle espèce de créature. (Houellebecq, 1998, p. 53)

Ensuite, être femme s'avère être une fatalité avilissante, scellée d'un trait biologique natif: «Il se peut, sympathique ami lecteur, que vous soyez vous-même une femme. Ne vous en faites pas, ce sont des choses qui arrivent.» (Houellebecq, 1994, p. 9)

Sous forme de devinette, la femme se trouve complètement réifiée, il n'en subsiste que sa fonction d'accouplement. Elle est représentée par une image réductrice, caricaturale qui verse dans l'humiliation la plus pure. Cette image survient en plus, dramatisée sous forme de dialogue empreint d'une oralité très familière, qui semble transmettre la vision du monde de la majorité de la société: "-Tu sais comment on appelle le gras qu'y a autour du vagin ? -Non. -La femme.» (Houellebecq, 2005, p. 18)

\section{Le métissage comme phénomène douloureux: le retour de l'autre}

Les bouleversements que le monde a connus tout au long du siècle dernier, ont montré que le nationalisme, la religion et les particularismes culturels représentent une réalité humaine enracinée dans les profondeurs de la condition humaine. Prendre en compte le phénomène religieux comme un critère de reconnaissance et d'identité, est pourtant, incompatible avec une pensée européenne, qui a procédé, avec ténacité, 
à la désaffectation de l'Église (Huntington, 2007, p. 6). L'idéologie de la mondialisation, présumée multiculturaliste et antiraciste, provoque et transfère les guerres entre nations en guerres à l'intérieur des nations (Zemmour, 2014, p. 253).

Des bribes racistes disséminées çà et là expriment clairement l'orientation raciste de Houellebecq et préparent le débat qui n'est jamais affronté de face. Il y a nécessairement deux mondes bien distincts, celui des occidentaux et celui des non occidentaux. Houellebecq n'épargne pas les occidentaux, mais le racisme, à leur égard reste modéré. En effet, les asiatiques ont une autre structure mentale (Houellebecq, 2005, p. 46) incompatible avec celle des Occidentaux, les Orientaux exagèrent (Houellebecq, 2005, p. 64), les Brésiliens sont des abrutis et les Chinois sont reconnaissables à leur saleté (Houellebecq, 2005, p. 20).

Les musulmans sont particulièrement appréhendés par une énonciation double et mise en abîme, toujours par l'intermédiaire d'un interlocuteur musulman, qui s'exprime en discours direct afin de rapporter plus de crédit à sa critique (Houellebecq, 2001, p. 64). «Des tas. Des gros tas de graisse informes qui se dissimulent sous des torchons. Dès qu'elles sont mariées, elles ne pensent plus qu'à manger. Elles bouffent, elles bouffent, elles bouffent! » (Houellebecq, 2001, p. 30).

Aïcha, femme de ménage d'origine nordafricaine, illustre bien la dissension dans la coexistence de deux systèmes de valeurs Islam / Occident. Ils traitent Aicha de prostituée parce qu'elle défie leur domination et refuse de vivre selon les traditions.

Elle dévoile la supercherie des membres de sa famille qui, théoriquement, obéissent aux préceptes islamiques et se croient plus proches de Dieu que les autres, paradoxalement, ils se soûlent bien que l'alcool soit prohibé par la religion :

«Non seulement ils sont pauvres, mais en plus ils sont cons. Il y a deux ans, mon père a fait le pèlerinage de La Mecque ; depuis, il n’y a plus rien à en tirer ». [...] Mes frères, c'est encore pire : ils s'entretiennent mutuellement dans leur connerie, ils se bourrent la gueule au pastis tout en se prétendant les dépositaires de la vraie foi, et ils se permettent de me traiter de salope parce que j'ai envie de travailler plutôt que d'épouser un connard dans leur genre. (Houellebecq, 2001, p. 30)

Enfin les musulmans sont présentés s'acharnant sur la sexualité comme la priorité ultime dans la vie et focalisent spécifiquement leur intérêt sur leur valeur érotique.

Houellebecq trouve que le métissage culturel empêche une cohabitation parfaite de différents sujets sociaux dont la vision du monde freine leur intégration totale. La religion, génératrice d'innombrables clivages sociaux, rend toute intégration sociale impossible, tant l'hétérogénéité/l'hybridation cultuelle s'avère insurmontable (Hladki, 2015, p. 9). Pourtant, tout en étant racistes, les personnages rêvent d'avoir des rapports 
sexuels avec des filles d'origines nord-africaines (Houellebecq, 2001, p. 30). Il s'agit dans ce cas d'un rapport de dominant/dominé réconfortant qui dépasse le racisme biologique homme/femme à la discrimination de race.

Dans ce sens, la notion du racisme, autrement dit, l'existence quasi mythique, d'une différence biologique ou autre (Encyclopédie Universelle, s.d.), tire sa justification de la peur. Le racisme porte atteinte directe à l'instinct de vie et le sentiment de sécurité. Or les pulsions instinctives comme la peur, déclenchent l'animalité et l'agressivité. Refuser l'Autre et s'affirmer soi-même contre cet Autre en le brutalisant, deviennent ainsi une réaction instinctive à la menace, donc refus et affirmation justifiés, le racisme du narrateur contre les autres devient légitime:

«C'est vrai que dans mon quartier, c'est plus les Blancs qui font la loi... intervint Lionel sans nécessité apparente.

—Exactement! approuva Robert avec force. Vous avez peur, et vous avez raison d'avoir peur. Je prévois pour les années à venir une augmentation des violences raciales en Europe; tout cela se terminera en guerre civile, dit-il en écumant légèrement ; tout cela se réglera à la Kalachnikov. » [....] Je suis un Occidental, mais je peux vivre où je veux, et pour l'instant c'est encore moi qui ai le fric. (Houellebecq, 2001, p. 29)

L'Autre, ici la faction armée du fondamentalisme islamique, impose sa vision; les attentats perpétrés ont pour objectif de rappeler à la probité et à la règlementation religieuse les « égarés » échappés dans les zones touristiques : «Deux personnes étaient mortes. Une troisième avait les jambes sectionnées et la moitié du visage arraché ; elle resterait mutilée et aveugle [...] (le coup) déchiquetant une femme d'une cinquantaine d'années » (Houellebecq, 1994, p. 12).

Un autre objectif des attentats est d'exécuter, un châtiment préliminaire icibas, pour contrer la prostitution et les élans sexuels des apostats occidentaux :

La jeune fille avait été lapidée, on s'était acharné sur elle avec une violence extrême; la peau avait éclaté de partout, son corps n'était plus qu'une boursouflure à peine reconnaissable. L'Allemand avait été égorgé et châtré sa verge et ses testicules étaient enfoncés dans sa bouche. (Houellebecq, 2001, p. 79)

La légitimité de l'agression contre cet Autre, qui se fondait dans le passé, sur la différence biologique, ou l'exclusion théologique ou politique comme au moyen âge, cède la place aujourd'hui à l'exclusion culturelle, B. Noël explique bien cette distinction : "Le racisme, c'est un regard qui vous classe sans appel. Qu'importe où il vous range, il a ouvert la différence et rien ne l'efface plus » (Encyclopédie Universelle, s.d.). 
Par sa différence, sa non-référentialité et son étrangeté culturelle, cet Autre (les ex-colonisés et les immigrés) fait encore plus peur lorsqu'il vient avec sa violence, menacer le moi occidental dans son écosystème culturel. «Les origines des gens font partie de leur personnalité, il faut en tenir compte, c'est évident ». (Houellebecq, 2001, p. 51)

L'argumentation raciste de Houellebecq se décline en xénophobie. Son raisonnement par l'absurde se développe à la dimension d'une fiction entière; Soumission figure ainsi, comme une fable à rebours de l'hybridation et du métissage. Partant de l'hypothèse de l'avènement d'un président musulman pour la France, Houellebecq procède à l'islamisation de toute la France et explicite les changements démographiques, géopolitiques et culturels qui amèneraient la fin de la nation française, telle qu'elle est connue le long de l'Histoire. Le système religieux s'impose avec ses vraies valeurs, dont les bienfaits sont incontournables, en effet, il a remporté le consensus politique, économiquement, il a remédié au chômage ; sur le plan social, sa doctrine a diminué l'exacerbation du désir, en éliminant toute séduction féminine ostentatoire. Enfin, la polygamie dissipe toute frustration masculine et alimente les fantasmes endormis.

Effectivement, Houellebecq écrivain semble dire à son personnage emblématique, François, dont le nom est symbolique par sa nationalité, qu'il faut se soumettre, mais Houellebecq blanc, chrétien, occidental, semble dire aux politiciens français qu'il faut réagir.

Nous découvrons que toute une série de choix paradigmatiques d'oppositions binaires opérés dans l'ordre des signifiants, fonctionnent de la même manière et procèdent à une mise à nu critique, d'une discrimination qui varie sur les divers traits allant du biologique (couleur), à l'économique, au cultuel (religieux) au sein du monde marchand. La problématique du racisme / distinction hiérarchisée, apparaît comme un axe majeur et ses déclinaisons présuppositionnelles se déploient dans l'œuvre de Houellebecq sous forme de microphénomènes ayant un rapport d'opposition :

- Dans le domaine de la sexualité : jeunes/âgés, male/femelle, homme / femme, beau/laid

- Dans le domaine de l'économie : Occident/Orient, Sud/Nord, pauvre/ riche

- Dans le domaine biologique : race blanche/race non blanche néoespèce (espèce humaine manipulée)/ espèce humaine.

- Dans le domaine culturel et religieux : occidentaux/orientaux, civilisés/barbares, chrétiens/musulmans

Bien qu'il dénonce constamment dans ses romans, un Occident décadent et inhumain, Houellebecq à travers son narrateur, favorise le racisme contre l'autre, ren- 
dant ainsi la visée humaniste de ses romans très discutable. Cela permettra effectivement, d'explorer plusieurs aspects de la complexité des relations intercommunautaires et de déterminer dans quelle mesure sa littérature considère les interférences ethnoreligieuses comme une menace pour la société et pour le fonctionnement optimal de la vie publique.

Dans le jeu du dit et du non-dit, la transparence s'avère complètement absente laissant la place à une opacité consciente et volontaire à visée argumentative. Les problématiques soulevées par Houellebecq révèlent la religion comme une source non négligeable de différenciation sociale, défavorable à une intégration totale d'une population donnée. Si l'hybridation comme nouveau paradigme d'imagination dialogique et d'harmonie culturelle, essaie de voiler la présence violente du racisme et des inégalités structurelles en matière de sexe, de classe, de préférence sexuelle et d'identité culturelle, Houellebecq à travers ses multiples narrateurs interposés, amène la confusion aux esprits. La grande force de son argumentation, réside dans le fait qu'il ne pose pas d'idéologie localisable, mais qu'il orchestre un certain rapport, favorable ou non favorable, à l'idéologie, et plus encore à la pensée de l'autre, qu'il présente toujours, comme suspectée d'erreur et d'absurdité, et par là, complètement discréditée.

\section{RÉFÉRENCES BIBLIOGRAPHIQUES}

Appadurai, A. (2001). Après le colonialisme:les conséquences culturelles de la globalisation. Payot.

Badaoui-El-Najjar, Z. (2016). Transparence et outrance dans l'cuvre de Michel Houellebecq [Thèse doctorale]. Université du Liban.

Bartnik, M. (2015, 3 septembre). Comprendre la crise des subprimes en quatre questions simples. Le Figaro. https://www.lefigaro.fr/economie/le-scan-eco/explicateur/2015/09/03/2900420150903ARTFIG00126-la-crise-des-subprimes-en-quatre-questions.php

Bellemin-Noël, J. (1979). Vers l'inconscient du texte. PUF

Bridet, G. (2008). Michel Houellebecq et les montres molles. Littérature, 151(3), 6-20. https://doi. org/10.3917/litt.151.0006

Carrère, E. (2015, 6 janvier). Emmanuel Carrère sur Houellebecq:«Un roman d'une extraordinaire consistance romanesque ». Le Monde. http://www.lemonde.fr/livres/article/2015/01/06/ emmanuel-carrere-la-resistance-n-interesse-pas-houellebecq_4550129_3260.html? xtmc $=$ houellebecq\&xtcr $=3$

Chivallon, C. (2007). La quête pathétique des postcolonial studies ou la révolution manquée. Mouvements, 51(3), 32-39. https://doi.org/10.3917/mouv.051.0032

Coquet, J-C. (2007). Phusis et Logos, Une phénoménologie du Langage. Presses Universitaires de Vincennes (collection La Philosophie hors de soi).

Cros, E. (2006, 13 octobre). Spécificités de la sociocritique. La sociocritique d'Edmond Cros. https:// www.sociocritique.fr/?Specificites-de-la-Sociocritique-d-Edmond-Cros 
Encyclopaedia Britannica Online. (s.d). Jeff Koons. In Encyclopaedia Britannica. Récupéré le 22 mai 2015 sur http://www.britannica.com/EBchecked/topic/761332/jeff-koons

Encyclopédie Universelle. (s.d.). Racisme. In Encyclopédie Universelle. http://encyclopedie_ universelle.fracademic.com/17458/RACISME

Hładki, P. (2015). Pluralité des cultures dans l'œuvre de Michel Houellebecq et de Jerzy Pilch. Folia Litteraria Romanica, 1(9), 155-162. https://hal.archives-ouvertes.fr/hall-01183999

Houellebecq, M. (2010). Extension du domaine de la lutte. Flammarion.

Houellebecq, M. (2010). La carte et le territoire. Flammarion.

Houellebecq, M. (2001). Plateforme. Flammarion.

Houellebecq, M. (2016). La possibilité d'une île. Flammarion.

Houellebecq, M. (2015). Soumission. Flammarion.

Houellebecq, M. (2000). Les Particules élémentaires. 2000. J'ai Lu.

Huntington, S. (2007). Le choc des civilisations. Éditions Odile Jacob.

Hutnyk, J. (2005). Hybridity. Ethnic and Racial Studies, 28(1), 79-102, https://doi.org/10.1080/ 0141987042000280021

Iablokov, A. V. (s.d). Struggle for Life. The Great Soviet Encyclopedia, 3rd Edition. (1970-1979). https://encyclopedia2.thefreedictionary.com/Struggle+for+Life

Ippolito, P. (2001-2002). Génération Houellebecq:exhibition outrancière de l'abîme intime [Travail de candidature de maitrise]. Lycée Technique d'Esch-sur-Alzette. https://www.yumpu.com/ $\mathrm{fr} / \mathrm{document} / \mathrm{read} / 16912862 /$ extension-du-domaine-michel-houellebecq

Jodelet, D. (2006). Place de l'expérience vécue dans le processus de formation des représentations sociales in V. Hass Les savoirs du quotidien. Transmissions, Appropriations, Représentations (pp. 235-255.) Les Presses universitaires de Rennes (Collection: Didact - Psychologie sociale http://classiques.uqac.ca/contemporains/jodelet_denise/place_experience_ processus/place_experience_processus.html

Joffrin, L. (2015, 2 janvier). «Soumission», Le Pen au Flore. Libération. http://wwwwliberation.fr// livres/2015/01/02/le-pen-au-flore_1173182

Lançon, P. (2015, 2 janvier). Un dandy de grand chemin. Libération. https://next.liberation.fr/ livres/2015/01/02/un-dandy-de-grand-chemin_1173185

Lipovetsky, Gilles. (2006). Le bonheur paradoxal. Essai sur la société d'hyperconsommation. Gallimard (Collection NRF Essais).

Machado Da Silva, J. (2010). Traduction et communication : la reliance des cultures. Hermès, La Revue, 56(1), 181-187. https://doi.org/10.4267/2042/38623

Ministère de l'Économie des Finances et de la Relance (s.d). John Maynard Keynes. http://www. economie.gouv.fr/facilleco/john-maynard-keynes

Sauvaire, M. (2012). Hybridité et diversité culturelle du sujet: des notions pertinentes pour former des sujets lecteurs?, Litter@ Incognita, 4. http://blogs.univ-tlse2.fr/littera-incognita-2 /2016/02/16/numero-4-2011-article-3-ms/

Schneidermann, D. (2015, 4 janvier). Si Houellebecq n'était pas Houellebecq ... Libération. http:// www.liberation.fr/ecrans/2015/01/04/si-houellebecq-n-etait-pas-houellebecq_1173765

Senghor, L.-S. (1964). Liberté 1. Négritude et humanisme. Seuil.

Todorov, T. (2008). La peur des barbares, Au-delà du choc des civilisations, Robert Laffont. 
Trétiack, P. \& Sirvem, S. (2007). Limite vulgaire. Stock (Hors collection littérature française).

Vesterberg, J. (2003). The Sexual Political Economy of Postmodernity; An Introduction to Critical Theory in the Works of Michel Houellebec. http://www.geocities.ws/grostock/ acwriting/Houellebecq_crtique.pdf

Zappi, S. (2013, 11 octobre). La Marche des beurs veut entrer dans l'histoire de France. Le Monde. https://www.lemonde.fr/societe/article/2013/10/11/la-marche-des-beurs-veut-entrerdans-I-histoire-de-france_3494031_3224.html\#OwzDcFREYf1ylmzh.99

Zemmour, E., (2014). Le suicide français. Les quarante années qui ont défait la France. Éditions Albin Michel. 